Les nombres $C$ que j'ai appelés les caráctéristiques de chaque système se trouvent être presqu'exactement proportionnels à la racine cubique de la densité $d$ de l'astre central. Il en résulte immédiatement que la Lune ne peut être que le quatrième satellite de la Terre: il faut en outre tenir compte de ce que la loi (2) ne s'applique que dans l'écliptique ou l'équateur d'un système planétaire. Dans le tableau 2 la correspondance entre le système solaire et celui de Saturne ressort d'une manière remarquable: elle est due à ce fait que l'axe de l'écliptique fait le même angle de $28^{\circ}$ avec la direction de translation vers l'apex et avec l'axe de Saturne.

$x_{11}$ donne la masse maxima (Jupiter-Titan), $x_{8}$ la masse secondaire maxima (Terre-Rhéa), $x_{9} x_{10}$ des astres très petits non encore découverts dans le système de Saturne. Les distances $x_{1} x_{2} x_{3}$ qui donnent les rayons des trois anneaux de Saturne correspondent à l'anneau zodiacal; $x_{4}=0.3386$ dans le système solaire est la distance-périhélie de la comète d'Encke où Backlund a déjà soupçonné la présence de matière produisant son accélération ${ }^{1}$ ). Les masses situées aux distances $x_{1} x_{2} x_{3} x_{4} x_{6}$ expliqueraient bien l'accélération de $38^{\prime \prime}$ par siècle du périhélie de Mercure et Newcomb a été amené à supposer de la matière entre Mercure et Vénus pour expliquer certaines inégalités de cette planète. Même en extrapolant dans la région rétrograde où la loi (2) théoriquement ne s'applique pas, on trouve pour la distance de Neptune 33.1 au lieu de la valeur 38.8 très inexacte que donne la loi de Bode et dont Leverrier s'est servi pour calculer la masse de Neptune.

Les deux systèmes dont l'axe présente le plus de symétrie par rapport à l'écliptique (Uranus et Jupiter) sont aussi ceux où la précision de l'application de la loi (2) est la plus grande. L'écart entre le calcul et l'observation

Paris, I 9 I 3 Mars.

(15.74-1 15.057 ) pour la distance du satellite III de Jupiter correspond précisément à la loi de libration des satellites I, II, III $\left(n_{1}+2 n_{3}=3 n_{2}\right)$ démontrée par Laplace. Enfin un fait général ressort du tableau II : une poussière d'astéroides semble toujours exister au-dessous de Jupiter, de Titan, de Titania, de la Lune, des gros satellites de Jupiter.

Conclusions. N'ayant en vue dans cet article que de marquer la puissance d'investigation de la méthode inductive en Cosmogonie, je renvoie le lecteur aux théories par lesquelles j'ai démontré les lois empiriques ( 1 ) et $(2)$ dans mon "Essai de Cosmogonie tourbillonnaire" (Paris, I9I I). Mais plusieurs conclusions suggestives peuvent être déjà tirées de l'étude précédente au point de vue de la validité de certaines théories cosmogoniques.

Si la formule (I) des rotations exprime bien une loi de la Nature, il faut en conclure que jamais les marées n'ont eu dans le passé l'importance que leur a attribuée Darwin et que leur influence sur les rotations a été, comme le pense $T$. See, contrebalançée par des actions contraires. D'ailleurs si la Lune est le quatrième satellite de la Terre, comme l'indique le tableau II, l'action de marée des satellites $x_{1} x_{2} x_{3}$ (avant que ceux-ci ne soient absorbés soit par la Lune soit par la Terre) a dû contrarier et détruire en grande partie celle de la Lune.

En second lieu les nombreuses et précises vérifications de la loi de distribution (2) semblent bien l'imposer comme une loi de la Nature. Alors la théorie de $T$. See de l'évolution cosmique par la capture croule par la base: car la probabilité pour que des phénomènes de capture essentiellement dus au hasard aient donné lieu à une loi de distribution qui est la même dans des plans aussi différents que ceux de l'écliptique et des équateurs de Saturne et d'Uranus, est infiniment petite.

Emile Belot.

1) La correspondance entre les deux systèmes s'étend même aux valeurs des masses (première et dernière colonne du tableau II) quì sont exprimées par des nombres très voisins pour les mémes valeurs de $n$. On aurait pu de là inférer que la masse de Titan adoptée jusqu'ici était trop faible, ce que $H$. Struve a démontré récemment.

\title{
Osservazioni di Comete e di Pianeti
}

fatte all'Osservatorio Astronomico della R. Università di Padova. Da A. Antoniazzi.

\begin{tabular}{l|l|l|l|l|l|l|l|l|l|}
\hline I9 I2 & T.m. Padova & $\Delta \alpha$ & $\Delta \delta$ & Cf. & $\alpha$ app. & $\log p \cdot \Delta$ & $\delta$ app. & $\log p \cdot \Delta \mid \operatorname{Red}$. ad l. app. & $*$ \\
\hline
\end{tabular}

\section{Cometa rgra a (Gale).}

\begin{tabular}{|c|c|c|c|c|}
\hline \multirow[t]{10}{*}{ Ott. } & 9 & $6^{\mathrm{h}} 5^{8^{\mathrm{m}} 27^{\mathrm{s}}}$ & $+3^{\mathrm{m}} 17^{\mathrm{s}} \cdot 53$ & $-\circ^{\prime} 53^{\prime \prime} \mathrm{I}$ \\
\hline & I I & $6 \quad 50 \quad 32$ & +343.69 & -10 \\
\hline & 12 & $\begin{array}{lll}6 & 32 & 56\end{array}$ & +258.22 & t- 0 III.I \\
\hline & 13 & 62622 & + I 44.65 & $+\circ 14.0$ \\
\hline & I 4 & $\begin{array}{lll}6 & 20 & 3^{8}\end{array}$ & +412.26 & +610.9 \\
\hline & 15 & $\begin{array}{lll}6 & 142\end{array}$ & $-\mathrm{I} 25 . \mathrm{I} 3$ & $-\circ 41.0$ \\
\hline & 16 & $\begin{array}{lll}6 & \text { I } 8 & 3^{8}\end{array}$ & -05 I.6I & +358.9 \\
\hline & 20 & $\begin{array}{lll}6 & \text { I } 3 & 25\end{array}$ & to 34.44 & +333.5 \\
\hline & 20 & $\begin{array}{lll}6 & 13 & 25\end{array}$ & -023.36 & -5 \\
\hline & 28 & 254 & -242.57 & +321.0 \\
\hline & 7 & $\begin{array}{lll}5 & 49 & 43\end{array}$ & $-0 \quad 14.20$ & - 022.3 \\
\hline & 10 & $\begin{array}{lll}5 & 5^{6} & 5^{1}\end{array}$ & $7 \cdot 5 \mathrm{I}$ & -0 \\
\hline
\end{tabular}

\begin{tabular}{|c|c|c|}
\hline 8,8 & \multicolumn{2}{|c|}{$15^{\mathrm{h}} 3^{6^{\mathrm{m}}} 34^{\mathrm{s}}: 83$} \\
\hline 6,6 & $15 \quad 39$ & $5^{8.85}$ \\
\hline 10,10 & I 54 I & 32.69 \\
\hline I0, 10 & I 543 & 2.59 \\
\hline 7,7 & I 544 & 28.05 \\
\hline 10,10 & 1545 & 49.83 \\
\hline IO, IO & I 547 & 8.84 \\
\hline Io, ro & I 55 I & 48.91 \\
\hline IO, IO & I 55 I & 48.92 \\
\hline I O, IO & I $5 \quad 59$ & 24.76 \\
\hline ro, Io & 167 & $37 \cdot 34$ \\
\hline 10,10 & I 6 Io & 5.62 \\
\hline
\end{tabular}

\begin{tabular}{l|rrr}
$9.5^{8} 5$ & $+3^{\circ}$ & $54^{\prime}$ & 24.5 \\
9.584 & +6 & 6 & 31.2 \\
9.571 & +7 & 9 & 54.2 \\
9.569 & +8 & 12 & 26.4 \\
9.567 & +9 & 13 & 38.6 \\
9.564 & +10 & 13 & 29.1 \\
9.572 & +11 & 12 & 39.2 \\
9.584 & +14 & 55 & 15.1 \\
9.584 & +14 & 55 & 13.0 \\
9.612 & +21 & 30 & 18.4 \\
9.650 & +28 & 39 & 33.7 \\
9.668 & +30 & 40 & 38.7
\end{tabular}

\begin{tabular}{l|l}
0.782 & +1.05 \\
0.774 & +1.03 \\
0.766 & +1.02 \\
0.760 & +1.01 \\
0.754 & +0.99 \\
0.750 & +0.99 \\
0.745 & +0.97 \\
0.727 & +0.90 \\
0.727 & +0.90 \\
0.692 & +0.76 \\
0.658 & +0.56 \\
0.663 & +0.49
\end{tabular}

\begin{tabular}{l|r}
-7.2 & I \\
-6.8 & 2 \\
-6.7 & 3 \\
-6.6 & 4 \\
-6.6 & 5 \\
-6.5 & 6 \\
-6.2 & 7 \\
-6.1 & 8 \\
-6.0 & 9 \\
-6.2 & 10 \\
-7.7 & I I \\
-8.3 & I 2
\end{tabular}




\begin{tabular}{|c|c|c|c|c|c|c|c|c|c|c|}
\hline 19I 2 & T.m. Padova & $d \alpha$ & $\not \delta$ & Cf. & $\alpha$ app. & $\log p \cdot \Delta$ & $\delta$ app. & $\log p \cdot \Delta$ & Red. ad 1. app. & * \\
\hline
\end{tabular}

Cometa ig i 2 c (Borrelly).

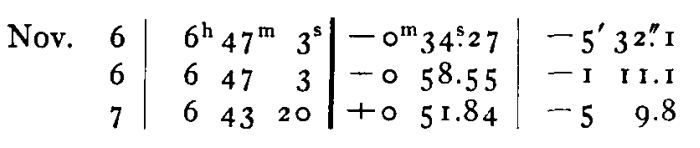

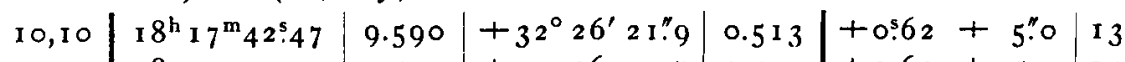

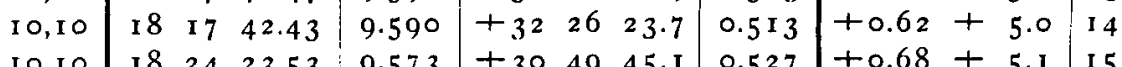

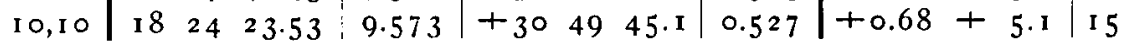

704 Interamia.

\begin{tabular}{rr|rrr|rr|rr} 
Gen. I0 & I0 & 49 & 28 & -0 & 1 & 7.05 & +2 & 25.2 \\
I & Io & 49 & 28 & - I & I 1.72 & -8 & 49.6 \\
Febb.20 & I 2 & 28 & 56 & -0 & 23.28 & +3 & 33.3 \\
22 & Io & 46 & 0 & -0 & 33.16 & +4 & 30.8 \\
Marz. 8 & 9 & 32 & 48 & +0 & I 6.99 & -2 & 41.6 \\
I I & 9 & I 5 & 32 & -0 & 9.72 & -2 & 8.6 \\
Apr. I I & 10 & 37 & 30 & +0 & 26.55 & -0 & 34.6 \\
I I & 10 & 37 & 30 & -0 & 26.83 & - I & 10.8
\end{tabular}

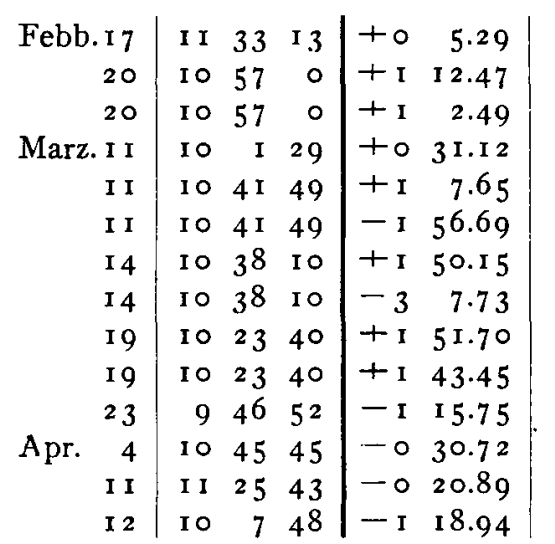

$\begin{array}{rr}+4 & 50.7 \\ +1 & 10.3 \\ +0 & 5.0 \\ +1 & 3.3 \\ +2 & 42.3 \\ -4 & 28.7 \\ +3 & 13 \cdot 7 \\ +3 & 40.5 \\ +6 & 28.4 \\ -1 & 0.6 \\ +2 & 3.3 \\ -2 & 35.0 \\ +1 & 8.1 \\ +0 & 38.7\end{array}$
\begin{tabular}{r|}
10,5 \\
10,5 \\
10,5 \\
5,5 \\
10,5 \\
10,5 \\
10,5 \\
10,5
\end{tabular}

\begin{tabular}{rrr|r}
8 & 23 & 57.80 & 9.3 \\
8 & 23 & 58.00 & 9.3 \\
7 & 49 & 31.22 & 9.4 \\
7 & 48 & 28.03 & 9.0 \\
7 & 43 & 9.49 & 8.9 \\
7 & 42 & 42.75 & 8.9 \\
7 & 49 & 27.11 & 9.5 \\
7 & 49 & 27.03 & 9.5
\end{tabular}
\begin{tabular}{l|rrr|r|r|r|r}
$9.376 n$ & +10 & 37 & 3.4 & 0.714 & $+1.01+3.7$ & 16 \\
9.376 & +10 & 36 & 59.7 & 0.714 & $+1.01+3.7$ & 17 \\
9.424 & +10 & 9 & 10.3 & 0.722 & $+1.23+2.2$ & 18 \\
9.058 & +10 & 9 & 30.1 & 0.706 & $+1.22+2.1$ & 19 \\
8.993 & +10 & 12 & 53.5 & 0.704 & $+1.05+2.2$ & 20 \\
8.951 & +10 & 13 & 26.5 & 0.704 & $+1.02+2.2$ & 20 \\
9.567 & +10 & 4 & 25.1 & 0.750 & $+0.59+2.5$ & 19 \\
9.567 & +10 & 4 & 26.5 & 0.750 & $+0.59+2.5$ & 18
\end{tabular}

354 Eleonora.

I0,5 | I0 48 I $9.89|9.209 \mathrm{n}|+$ I 5 I 7 I $5.9|0.653|+1.47-5.7 \mid 2$ r

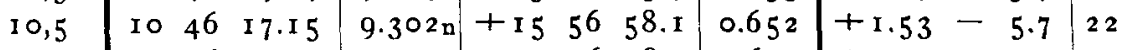

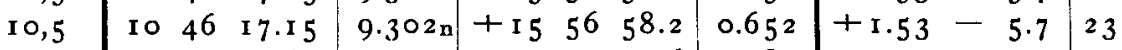

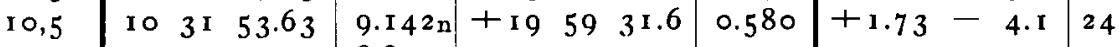

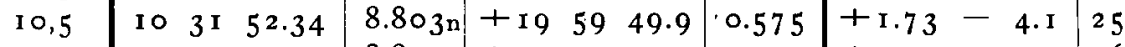
\begin{tabular}{l|lll|l|l|l|l|l|l}
10,5 & Io & $3 \mathrm{r}$ & 52.30 & $8.803 \mathrm{n}+19$ & 59 & 47.4 & 0.575 & $+1.74-4.3$ & 26
\end{tabular} \begin{tabular}{l|lll|l|l|l|l|l|l|l|l|}
10,5 & Io & 29 & 56.60 & $8.648 n$ & +20 & 29 & 37.8 & 0.566 & $+1.74-3.7$ & 27
\end{tabular}

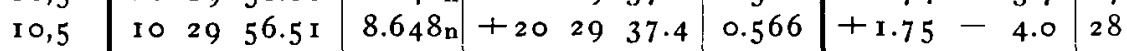

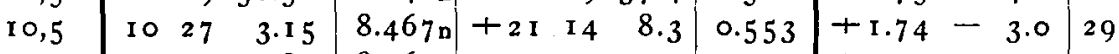

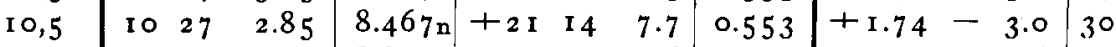

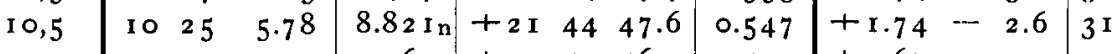

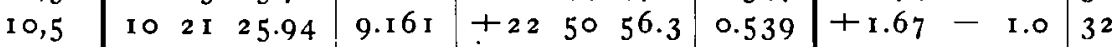
\begin{tabular}{l|lll|l|lll|ll|ll|l}
10,5 & I0 & $2 \mathrm{I}$ & 0.72 & $9.42 \mathrm{I}$ & +23 & II & 46.9 & 0.574 & +1.61 & -0.2 & 3.3
\end{tabular}

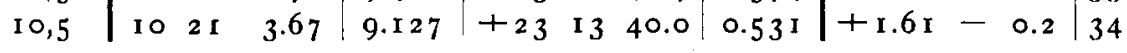

27 Euterpe.

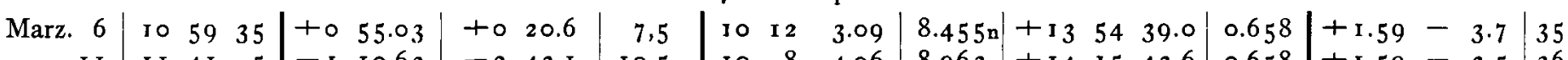

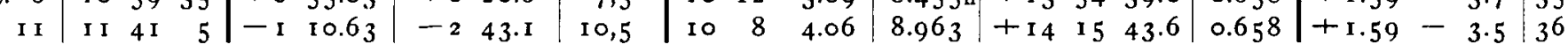

247 Eukrate.

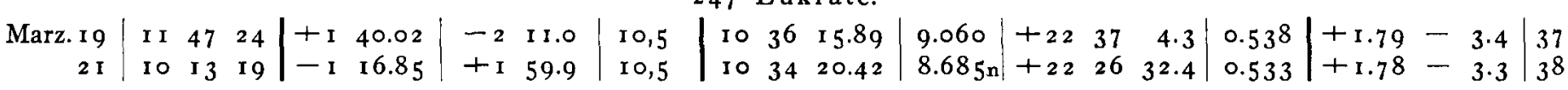

I 54 Bertha.

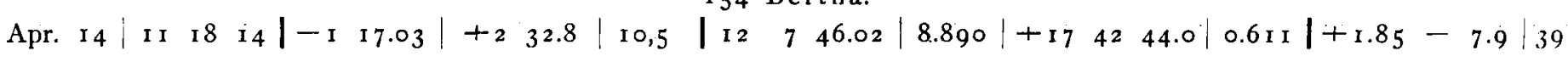
363 Padua.

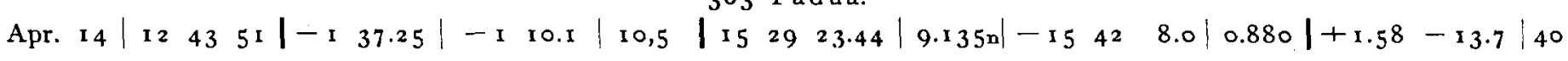
78 Diana.

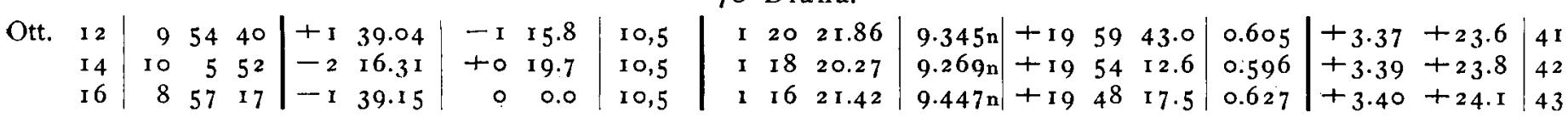
7 I Niobe.

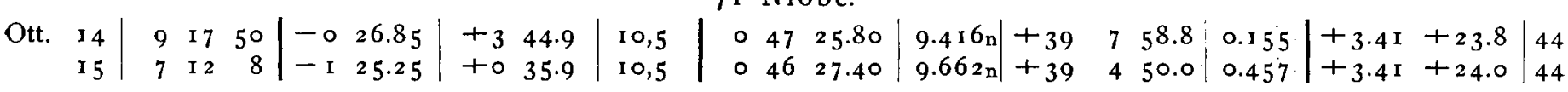
28 Bellona.

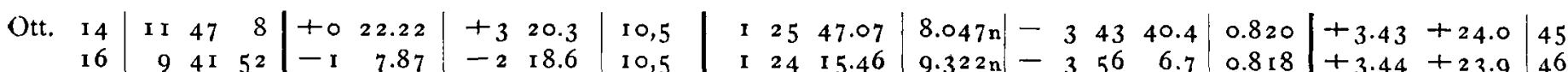


Stelle di confronto.

\begin{tabular}{|c|c|c|c|c|c|c|}
\hline$*$ & \multicolumn{3}{|c|}{$\alpha 1912.0$} & \multicolumn{3}{|c|}{$\delta$ I 9 I 2.0} \\
\hline I & I $5^{1}$ & $33^{n}$ & 16.25 & $+3^{\circ}$ & $55^{\prime}$ & 24.8 \\
\hline 2 & 15 & $3^{6}$ & I 4.13 & +6 & I 6 & $3^{8.2}$ \\
\hline 3 & I 5 & $3^{8}$ & 33.45 & +7 & 9 & 49.8 \\
\hline 4 & 15 & $4 \mathrm{I}$ & I 6.93 & +8 & 12 & 19.0 \\
\hline 5 & I 5 & 40 & 14.80 & +9 & 7 & $34 \cdot 3$ \\
\hline 6 & I 5 & 47 & I 3.97 & +10 & 14 & I 6.6 \\
\hline 7 & I 5 & 47 & 59.48 & $+\mathbf{I} \mathbf{I}$ & 8 & 46.5 \\
\hline 8 & I 5 & $5^{I}$ & I 3.57 & +14 & $5^{\mathrm{I}}$ & $47 \cdot 7$ \\
\hline 9 & I 5 & $5^{2}$ & I I. $3^{8}$ & +15 & 0 & 25.6 \\
\hline IO & I 6 & 2 & 6.57 & $+2 I$ & 27 & 3.6 \\
\hline I I & I 6 & 7 & 50.98 & +28 & 40 & $3 \cdot 7$ \\
\hline I 2 & I 6 & 9 & 57.62 & +30 & 40 & 53.6 \\
\hline I 3 & 18 & I 8 & I 6.12 & +32 & $3 I$ & 49.0 \\
\hline I 4 & I 8 & I 8 & 40.36 & +32 & 27 & 29.8 \\
\hline 15 & I 8 & 23 & 31.01 & +30 & 54 & 49.8 \\
\hline 16 & 8 & 24 & I 3.84 & +10 & 34 & 34.5 \\
\hline I 7 & 8 & 25 & $8.7 \mathrm{I}$ & +10 & 45 & 45.6 \\
\hline I 8 & 7 & 49 & 53.27 & +10 & 5 & 34.8 \\
\hline I 9 & 7 & 48 & 59.97 & +10 & 4 & 57.2 \\
\hline 20 & 7 & 42 & 51.45 & +10 & I 5 & 32.9 \\
\hline $2 \mathrm{I}$ & IO & 48 & I 3.13 & + I 5 & I 2 & 30.9 \\
\hline 22 & IO & 45 & $3 . \times 5$ & +15 & 55 & $53 \cdot 5$ \\
\hline 23 & IO & 45 & 13.13 & + I 5 & $5^{6}$ & 58.9 \\
\hline
\end{tabular}

Padova, I9 3 Aprile.

\begin{tabular}{|c|c|c|c|c|c|c|}
\hline$*$ & \multicolumn{3}{|c|}{$\alpha$ I 912.0} & \multicolumn{2}{|c|}{$\delta 1912.0$} & Autorità \\
\hline 24 & IO & $3 x^{1}$ & $\mathrm{~m}_{20} \mathrm{~s}_{7} 8$ & $+19^{\circ} 5^{8}$ & 32.4 & Berl B 4062 \\
\hline 25 & Io & 30 & 42.96 & +1957 & I I.7 & $》 406$ I \\
\hline 26 & IO & 33 & 47.25 & $+20 \quad 4$ & 20.4 & $\gg \quad 407 \mathrm{I}$ \\
\hline 27 & ro & 28 & $4.7 \mathrm{I}$ & +2026 & 27.8 & 4047 \\
\hline 28 & Io & 33 & 2.49 & +2026 & 0.9 & 》4068 \\
\hline 29 & Io & 25 & $9.7 \mathrm{I}$ & $+2 \mathbf{I} 7$ & 42.9 & $403 \mathrm{I}$ \\
\hline 30 & Io & 25 & 17.66 & +21 I 5 & I I. 3 & $4032-33$ \\
\hline $3^{I}$ & IO & 26 & 19.79 & +2142 & 46.9 & $403^{8}$ \\
\hline 32 & Io & $2 \mathrm{I}$ & 54.99 & +2253 & $32 \cdot 3$ & Bo $\mathrm{VI}+23^{\circ} 2225$ \\
\hline 33 & 10 & $2 \mathrm{I}$ & 20.00 & +2310 & 39.0 & Berl B 4009 \\
\hline 34 & IO & 22 & 21.00 & +23 I 3 & I. 5 & $\gg \quad 4015$ \\
\hline 35 & IO & I I & 6.47 & +1354 & 22.1 & $\operatorname{Lp} z I_{3976}$ \\
\hline 36 & IO & 9 & I 3.10 & $+\mathrm{I} 4 \mathrm{I} 8$ & 30.2 & $\gg 3970$ \\
\hline 37 & Io & 34 & 34.08 & +2239 & I 8.7 & Berl B 4073 \\
\hline $3^{8}$ & 10 & 35 & 35.49 & +2224 & 35.8 & Bo VI $+22^{\circ} 225^{6}$ \\
\hline 39 & I 2 & 9 & 1.20 & +I 740 & I 9.1 & Berl A ${ }_{4623}$ m. p. \\
\hline 40 & 15 & 30 & $59.1 \mathrm{r}$ & - I 540 & 44.2 & Wa 5713 \\
\hline $4 \mathrm{I}$ & 1 & 18 & $39 \cdot 45$ & +200 & 35.2 & Berl A 395 m. p. \\
\hline 42 & I & 20 & 33.19 & + I9 53 & 29.1 & $\gg \quad 404$ \\
\hline 43 & I & 17 & 57.17 & +1947 & 53.4 & $\gg \quad 390$ \\
\hline 44 & $\circ$ & 47 & 49.24 & +393 & $5^{0.1}$ & $\mathrm{Lu}_{33} 8$ \\
\hline 45 & I & 25 & $2 \mathrm{I} .42$ & -347 & 24.7 & Strb $34 \mathrm{I}$ \\
\hline 46 & I & 25 & 19.89 & -354 & $\mathrm{I} 2.0$ & $» 340$ \\
\hline
\end{tabular}

Comètes observées à Genève, à l'Equatorial de 10 pouces.

\begin{tabular}{l|l|l|l|l|l|l|l|l|l|}
\hline I912-I3 T.m. Genève & $\Delta \alpha$ & $\Delta \delta$ & Cp. & $\alpha$ app. & Parall. & $\delta$ app. & Parall. & Red.ad l.app. & $*$ \\
\hline
\end{tabular}

Comète I 9 I $2 \mathrm{c}$ (Borrelly).

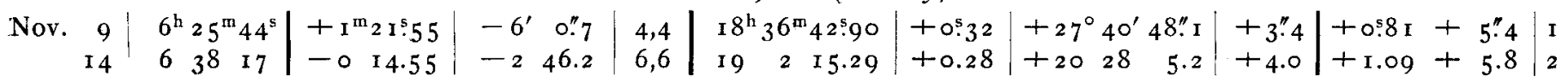
Comète I91 2 a (Gale).

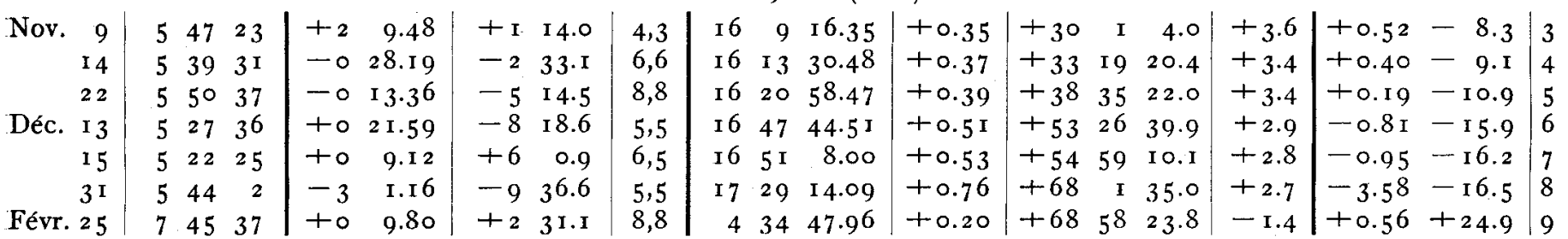

Positions moyennes des étoiles de comparaison.

\begin{tabular}{|c|c|c|c|c|c|c|c|}
\hline * & $\alpha$ I 9 I 2.0 & $\delta$ I912.0 & Autorité & * & $\alpha$ & $\delta$ & Autorité \\
\hline $\mathbf{I}$ & I $8^{\mathrm{h}} 35^{\mathrm{m}} 20^{\mathrm{s}} \cdot 54$ & $+27^{\circ} 46^{\prime} 43^{\prime \prime} 4$ & Cbr E. 9099 & 6 & $\begin{array}{c}\text { I } 912.0 \\
\text { I } 6^{\mathrm{h}} 47^{\mathrm{m}} 23^{\mathrm{s}} \cdot 73\end{array}$ & $\begin{array}{c}1912.0 \\
+53^{\circ} 35^{\prime} \times 4.4\end{array}$ & Cbr M. $5 \circ 87$ \\
\hline 2 & $19 \quad 2 \quad 28.75$ & +203045.6 & Berl B 6838 & 7 & I $6 \quad 50 \quad 59.83$ & +545325.4 & $\gg \quad 5103$ \\
\hline 3 & $\begin{array}{lll}\text { I } 6 & 7 & 6.35\end{array}$ & $+29595^{8.3}$ & Cbr E. $75^{1} 8$ & 8 & I 732 I 8.83 & +68 I I $28 . \mathrm{I}$ & $f$ Drac. (B. J. I 9 I 2$)$ \\
\hline 4 & I6 $13 \quad 58.27$ & $+3322 \quad 2.6$ & Kü 72 I 7 & & 1913.0 & I 913.0 & \\
\hline 5 & 1621 I 1.64 & $+38 \quad 40 \quad 47.4$ & Lu 6733 & 9 & $434 \quad 37.60$ & +685527.8 & Chri 757 \\
\hline
\end{tabular}

Remarques. La Comète r9izc (Borrelly) se présente comme une nébulosité faible avec un soupçon de noyau. Les pointés sont rendus difficiles surtout en $\alpha$ à cause de l'éclairage des fils. Diamètre total 2 à 3 minutes d'arc. La Comète Ig I a ( Gale) assez brillante en novembre, diminue peu à peu d'éclat et se trouve au 25 Févr. I 9 I 3 à la limite de visibilité. Elle a toujours possédé un noyau stellaire assez prononcé et visible encore lors de la dernière observation. Genève, 19 3 Févr. 28. F. Pidoux. 